\title{
Comparative in vitro analyses of recombinant maize starch synthases SSI, SSIIa, and SSIII reveal direct regulatory interactions and thermosensitivity
}

Binquan Huang ${ }^{\mathrm{a}, 1}$, Peter L. Keeling ${ }^{\mathrm{b}}$, Tracie A. Hennen-Bierwagen ${ }^{\mathrm{a}}$, Alan M. Myers ${ }^{\mathrm{a},{ }^{*}}$

${ }^{a}$ Roy J. Carver Department of Biochemistry, Biophysics, and Molecular Biology, Iowa State University, Ames, IA 50011, USA

${ }^{\mathrm{b}}$ Center for Biorenewable Chemicals, 1140K Biorenewables Research Laboratory, Iowa State University, Ames, IA 50011, USA

* Corresponding author. 2110 Molecular Biology Building, Ames, IA 50011, United States. Email address: ammyers@iastate.edu

${ }^{1}$ Present address. Department of Plant Sciences, University of Oxford, South Parks Road, Oxford, OX1 3RB, UK 


\begin{abstract}
Starch synthases SSI, SSII, and SSIII function in assembling the amylopectin component of starch, but their specific roles and means of coordination are not fully understood. Genetic analyses indicate regulatory interactions among SS classes, and physical interactions among them are known. The $\mathrm{N}$ terminal extension of cereal SSIII, comprising up to 1200 residues beyond the catalytic domain, is responsible at least in part for these interactions. Recombinant maize SSI, SSIIa, and full-length or truncated SSIII, were tested for functional interactions regarding enzymatic activity. Amino-terminal truncated SSIII exhibited reduced activity compared to full-length enzyme, and addition of the $\mathrm{N}$ terminus to the truncated protein stimulated catalytic activity. SSIII and SSI displayed a negative interaction that reduced total activity in a reconstituted system. These data demonstrate that SSIII is both a catalytic and regulatory factor. SSIII activity was reduced by approximately $50 \%$ after brief incubation at $45^{\circ} \mathrm{C}$, suggesting a role in reduced starch accumulation during growth in high temperatures. Buffer effects were tested to address a current debate regarding the SS mechanism. Glucan stimulated the SSIIa and SSIII reaction rate regardless of the buffer system, supporting the accepted mechanism in which glucosyl units are added to exogenous primer substrates.
\end{abstract}




\section{Keywords:}

Starch synthase

Starch structure

Maize

Endosperm

Thermosensitivity

List of Abbreviations: ADPGlc, ADP glucose; SS, starch synthase; DP, degree of polymerization; SSIII-HD, residues 770-1225 of full-length SSIII constituting the "SSIII homology domain"; SSIII-N, residues 90-769 of full-length SSIII constituting the $670 \mathrm{~N}$ terminal residues of the predicted mature protein as it exists in vivo, SSIII-CAT, residues 1226-1674 of full-length SSIII constituting the catalytic domain including the entire GT-B structural fold; SSIII-HDCAT, residues 770-1674 of full-length SSIII containing both the SSIII homology domain and the catalytic domain; SSIII-NHD, residues 90-1225 of full=length SSIII containing both the amino terminal domain and the SSIII-homology domain. 


\section{Introduction}

Starch, the main carbon reserve in plants, supports metabolic function owing in large part to the molecular architecture of its most abundant component amylopectin. A key feature of amylopectin structure is the length distribution of "linear", i.e., $\alpha(1 \rightarrow 4)$-linked, chains that are joined to each other by $\alpha(1 \rightarrow 6)$ branches. Linear chains are synthesized by starch synthases (SS) (EC 2.4.1.21) that catalyze addition of glucosyl units to the non-reducing end of an existing polymer using ADPglucose (ADPGlc) as the hexose donor [1-3]. All known genomes of chloroplast-containing organisms contain separate genes encoding five different SS classes designated granule-bound starch synthase (GBSS), SSI, SSII, SSIII, and SSIV [4]. In some species one or more of the genes have duplicated and taken on apparent tissue-specific functions, but in all green algae and land plants it appears that at least one gene for each class is active. Such evolutionary conservation suggests that each SS class provides a unique function. Some specific roles have been described, as follows $[2,5]$.

GBSS functions primarily in synthesis of the amylose component of starch, and the role of SSIV appears to be in granule initiation. The current study addresses SSI, SSII, and SSIII, which all participate in determining amylopectin structure. SSI appears to be primarily responsible for generating linear chains with degree of polymerization (DP) 8-12. SSII is thought to elongate such chains to achieve the full length present in the crystalline regions of mature starch grains, approximately DP 16-22. The substrate and product specificities of SSI and SSII overlap, and there appears to be competition between them in vivo for access to the acceptor substrate. The roles of SSIII appear to be more complex and to involve regulatory interactions separable from enzymatic activity, as discussed in a following section. 
The SS catalytic domain exhibits the conserved GT-B fold typical of glycogen synthase and other glycosyl transferases, comprising two separate Rossman folds designated as GT_1 and GT_5 (Pfam database IDs PF00534 and PF08323, respectively) [6, 7]. N terminal extensions beyond the catalytic domain, of unknown structure, are present in each SS class and are conserved to various extents (Fig. 1A). For example, the $\mathrm{N}$ terminal extensions of SSI from potato and maize are $22 \%$ identical over 78 aligned residues. SSIII contains the longest $\mathrm{N}$ terminal extension among these enzymes. This includes a conserved sequence of approximately 450 residues, designated as the SSIII homology domain (SIII-HD), that is adjacent to the catalytic domain (Fig. 1A). As a typical example, SIII-HD of maize and potato exhibit 57\% identity over 456 residues with no gaps in the alignment [8]. Both glucan binding and proteinprotein interaction functions have been attributed to the SSIII-HD domain [9-12]. Additional sequences of various lengths ranging from approximately 130 to 700 or more amino acids are present in the SSIII N terminus adjacent to SSIII-HD (Fig. 1A). This 700 amino acid region in maize SSIII, designated as SSIII-N, is conserved at a relatively low level in other monocot species, but not between monocots and dicots. Structural motifs are not predicted from $\mathrm{N}$ domain primary sequences. In at least some instances the $\mathrm{N}$ domain is involved in protein-protein interactions, as shown by a positive signal between part of SSIII-N and maize SSI in in vivo protein-protein interaction tests [11].

The current study utilized recombinant maize SSI, SSIIa, and SSIII, and fragments of SSIII, to address three aspects of SS function in amylopectin biosynthesis. The first aspect is regulatory interactions between different SS classes. SSIII has been implicated as a regulatory factor in addition to its biosynthetic role. SSIII mutation in maize or rice endosperm resulted in elevated rather than decreased total SS activity in cell extracts [13-15], 
and such mutations in Arabidopsis leaf caused elevated starch content rather than decreased amounts as predicted simply from loss of a catalytic activity [16, 17]. From these results SSIII appears to be a negative regulator of other SSs. Additional evidence for a regulatory role is that in cereals the effects of SSIII mutations on amylopectin structure are discontinuous with regard to the specific chain lengths that change in abundance $[15,18,19]$. In contrast, mutations affecting SSI or SSII result in alterations in continuous ranges of chain lengths [5]. SSI and SSII, therefore, appear to be involved in generation of linear glucan chains of certain length ranges, whereas SSIII appears to have multiple effects on amylopectin structure that cannot be explained only by substrate or product specificity. Finally, SSIII is a scaffold component of a high molecular mass complex that contains other starch biosynthetic enzymes [11, 12]. This report provides further evidence for regulatory interaction between specific SSs by recapitulating such effects in a reconstituted system.

SSs are also of interest because they appear to be sensitive to temperature to an extent greater than is typical for most enzymes [20-23]. Previous studies were performed with cell extracts containing undefined mixtures of SS classes, so the specific SSs that are hypersenstive to incubation temperature have not been defined. Such information is significant considering that when maize kernels develop under high temperature the yield is significantly decreased even when other conditions are optimal $[24,25]$. In the current study three recombinant maize SSs were tested individually for sensitivity to incubation at elevated temperatures.

Finally, the recombinant system was used to test for stimulation of SS activity by exogenous glucan polymer acting as an acceptor substrate. This commonly accepted mechanism was challenged in a recent publication suggesting that, rather than acting as a substrate, exogenous glucan alleviates an artifactual inhibition resulting from the buffer conditions standardly used for 
SS assays [26]. To test this proposal, recombinant SSs activities in the presence and absence of exogenous glucan were compared in various buffer systems.

\section{Materials and methods}

\subsection{Plasmid construction}

Table 1 documents the specific coding regions of maize SS enzymes expressed in E. coli, and indicates addition of $\mathrm{C}$ terminal His-tag or $\mathrm{N}$ terminal S-tag coding sequences. The $\mathrm{N}$ terminus of the expressed SSI protein matches the known in vivo sequence [27], whereas for SSIII and SSIIa the N termini were predicted from bioinformatic algorithms [28] because the native sequence has not yet been analyzed. Expression in E. coli utilized the pET-29 vector backbone (EMD Millipore) including the phage T7 promoter. Existing cDNA plasmids were used as PCR templates and the termini of the inserted regions were adjusted as needed to provide restriction sites within the sequences of the oligonucleotide primers. The nucleotide sequence of the inserts in all expression plasmids was determined (Table I). The amino acid sequence predicted by the SSI expression clone matched perfectly to that of the published cDNA (Genbank accession no. AF036891). All SSIII expression clones encoded amino acid sequences identical to those specified by the maize inbred B73 genome (gene model GRMZM2G141399), with the exception of a Val to Ala polymorphism at residue 1018. This polymorphism is presumed to be inconsequential with regard to SSIII function because a different inbred, W64A, encodes Ala at that position (Genbank accession no. JF273457). The amino acid sequence specified by the SSIIa expression clone was identical to that of the published cDNA (Genbank accession no. BT084561) except for a Leu to Pro polymorphism at position 534. 


\subsection{Protein expression and purification}

E. coli Rosetta2 (DE3) pLysS cells (EMD Millipore) were transformed with each expression plasmid. One positive clone was inoculated into $10 \mathrm{~mL}$ LB medium supplemented with kanamycin at $50 \mu \mathrm{g} / \mathrm{mL}$ (LB-Kan), and pre-cultures were grown at $37^{\circ} \mathrm{C}$ overnight. These were used to inoculate $500 \mathrm{~mL}$ fresh LB-Kan at $1 / 100$ dilution, and cultures were grown at $37^{\circ} \mathrm{C}$ to approximately $\mathrm{A}_{600}=0.6$. IPTG was added to $0.5 \mathrm{mM}$, and cultures were incubated at $28^{\circ} \mathrm{C}$ for 6 h. Cells were harvested by centrifugation, washed once in water, and cell pellets were frozen at $80^{\circ} \mathrm{C}$.

For purification of 6 XHis-tagged proteins cells were lysed by sonication in binding-wash buffer A (20 mM Tris- $\mathrm{HCl}, \mathrm{pH} 7.5,500 \mathrm{mM} \mathrm{NaCl}, 20 \mathrm{mM}$ imidazole) supplemented with $1 \mathrm{mM}$ PMSF, $5 \mathrm{mM}$ DTT, and $0.1 \mathrm{mg} / \mathrm{mL}$ lysozyme, followed by centrifugation at $10,000 \mathrm{rpm}$. Tagged proteins in the total soluble lysates were bound to Ni-NTA agarose (catalog no. R901-15, Invitrogen) equilibrated in binding-wash buffer $\mathrm{A}$, and the beads were then washed successively in binding-wash buffer A modified by addition of imidazole to $30 \mathrm{mM}, 60 \mathrm{mM}$, or $90 \mathrm{mM}$. Bound proteins were eluted in binding-wash buffer A modified by addition of imidazole to $250 \mathrm{mM}$. Eluted proteins were concentrated by centrifugal filtration (catalog no. UFC801024, EMD Millipore) and diluted into $50 \mathrm{mM}$ Tris- $\mathrm{HCl}, \mathrm{pH} 8.0,0.5 \mathrm{mM} \mathrm{NaCl}$. For long term storage at $-80^{\circ} \mathrm{C}$ glycerol was added to $25 \%(\mathrm{v} / \mathrm{v})$.

For S-tagged proteins, cells were lysed by sonication in binding-wash buffer B $(20 \mathrm{mM}$ Tris- $\mathrm{HCl}, \mathrm{pH} 7.5,150 \mathrm{mM} \mathrm{NaCl})$ supplemented as for the 6XHis-tag procedure. Recombinant products were bound to S-protein agarose (catalog no. 69704, EMD Millipore) equilibrated in binding-wash buffer B, and beads were washed extensively in the same buffer. SSI and SSIIa were cleaved from S-protein agarose using biotinylated thrombin according to 
manufacturer's instructions (catalog no. 69232-3, EMD Millipore). These were concentrated and stored as for 6 XHis-tagged proteins.

\subsection{SS activity assays and enzyme kinetic measurements}

SS activity was determined using the ADP Glo Kinase Assay Kit (catalog no. V6930, Promega) to quantify ADP generated during the reaction. Unless otherwise noted SS reactions were in $100 \mu \mathrm{L}$ volumes containing $100 \mathrm{mM}$ bicine- $\mathrm{NaOH}, \mathrm{pH}$ 8.0, $5 \mathrm{mM}$ EDTA, $0.5 \mathrm{mg} / \mathrm{mL}$ BSA, $5 \mathrm{mM}$ DTT, $10 \mathrm{mg} / \mathrm{mL}$ glycogen, and variable concentration of ADPGlc. Reactions were incubated at $30^{\circ} \mathrm{C}$ for $30 \mathrm{~min}$, then boiled for $2 \mathrm{~min}$ and centrifuged for 2 min at $12,000 \mathrm{rpm}$. Where noted the reaction buffer was modified by replacing the $100 \mathrm{mM}$ bicine buffer with either $10 \mathrm{mM}$ Tris- $\mathrm{HCl}$ or $10 \mathrm{mM}$ glycine- $\mathrm{NaOH}$, both at $\mathrm{pH}$ 8.0. To detect ADP, $5 \mu \mathrm{L}$ of the boiled SS reaction was transferred to a $100 \mu \mathrm{L}$ reaction in $40 \mathrm{mM}$ Tris- $\mathrm{HCl}$, $\mathrm{pH} 7.5,20 \mathrm{mM} \mathrm{MgCl} 2,01 . \%$ BSA, containing 5-10 $\mu \mathrm{L}$ of Kinase Detection Reagent from the assay kit. After 30 min incubation at room temperature the amount of photons released per unit time were quantified using a luminometer. Standard reactions containing 20-2000 pmol ADP were analyzed in parallel for each experiment, and these values generated a standard curve that was used to convert the photon count to the concentration of ADP in the SS reaction when it was boiled. The amount of enzyme added to each SS reaction was determined by Bradford assay. For enzyme bound to agarose beads, an equal volume of bed volume and $20 \mathrm{mM}$ Tris- $\mathrm{HCl}, \mathrm{pH} 7.5$ was mixed prior to the protein content assay.

Enzyme assays included approximately $100-500 \mathrm{ng}$ of recombinant protein. Preliminary assays with variable amounts of enzyme and $1 \mathrm{mM}$ ADPGlc established conditions in which enzyme was the limiting factor for initial velocity. For determination of ADPGlc Km values the concentration of that reactant was varied from $0.05-2.0 \mathrm{mM}$. Each enzyme reaction was 
repeated in triplicate. $\mathrm{Km}$ values were calculated individually from each replicate using double reciprocal plots and the separate values were averaged (Table 2). In addition, the initial velocities for each concentration of ADPGlc were averaged and these values displayed as double reciprocal plots (see Fig. 3 and Fig. 4).

For reconstitution experiments, different enzyme preparations were diluted to the same protein concentration then equal volumes were mixed. As controls, samples of one of the two components were boiled prior to mixing. Samples of the mixtures were then added to the SS reactions. Specific activity is reported based on the total protein concentration in the mixture, which was the same in all instances. Preliminary experiments determined the concentration range for each enzyme in which initial velocity was directly proportional to the amount of enzyme present. The total amount of enzyme in each reconstitution assay was adjusted to be within this linear range, to ensure that neither ADPGlc nor glycogen were limiting in the reactions.

\section{Results}

\subsection{Expression and purification of SSS and SSIII fragments}

Full-length SSI, SSIIa, and SSSIII were expressed in E. coli and affinity purified. The recombinant enzymes lacked the known or predicted plastid transit peptide, and were fused to either the 15 amino acid S-tag [29] at the N terminus (SSI and SSIIa) or the six amino acid 6XHis-tag [30] at the C terminus (SSIII) (Fig. 1B; Table 1). After expression from the phage T7 promoter, SSI and SSIIa were purified to near homogeneity by affinity to S-agarose, and fulllength SSIII was enriched to approximately $50 \%$ purity by binding to $\mathrm{Ni}^{++}$-agarose (Fig. 2). The molecular weights of recombinant SSI and SSIIa estimated by SDS-PAGE matched closely to 
the calculated values, whereas SSIII migrated at an anomalously high apparent value (Table 1, Fig. 2) as typical for this protein analyzed in endosperm extracts [14, 18].

Fragments of SSIII were obtained similarly (Fig. 1, Fig. 2). The catalytic domain SSIII-CAT comprises residues 1226-1674 denoted by the full length open reading frame, the conserved SSIII-homology domain SSIII-HD comprises residues 770-1225, and the cereal specific amino terminal domain SSIII-N comprises residues 90-769 (Table 1). Combinations of contiguous domains also were expressed, specifically SSIII-HDCAT and SSIII-NHD (Table 1, Fig. 1, Fig. 2). Both SSIII-N and SSIII-NHD migrated in SDS-PAGE at anomalously high apparent molecular weights, as did full length SSIII that also contains SSIII-N. The fragments lacking SSIII-N, specifically SSIII-CAT and SSIII-HDCAT, exhibited apparent molecular weights estimated by SDS-PAGE that matched the prediction from the open reading frame (Table 1, Fig. 2).

\subsection{Enzyme activities}

Purified protein fractions were assayed for SS activity using a bioluminescence measurement to quantify ADP released in the reaction of ADPGlc with glycogen as the acceptor substrate, to generate elongated glucan polymer. SSI and SSIIa were retained on S-agarose throughout the assay, whereas SSIII was free in solution after elution from $\mathrm{Ni}^{++}$-agarose. Specific activities measured in conditions of excess substrate were comparable between the three full length enzymes (Table 2). These assays were performed in the absence of citrate, even though in previous studies the non-physiological condition of $0.5 \mathrm{M}$ citrate was shown to stimulate the affinity of SSs for glycogen and increase the reaction rate [31-35]. Rates can be compared, however, between the various SS forms because all of them were assayed in identical conditions. Purified SSI, SSIIa and full-length SSIII were analyzed kinetically to determine the Km for 
ADPGlc (Table 2, Fig. 3). The results were again comparable between the three SS classes, and within the range of values previously reported for native and recombinant maize SSs [31-33].

To examine whether attachment to agarose beads through the S-protein/S-tag interaction at the $\mathrm{N}$ terminus affected enzyme activity, specific activities and $\mathrm{Km}$ values for ADPGlc were compared between SSI and SSIIa either free in solution after proteolytic release of the S-tag sequence or bound to agarose beads with the S-tag attached. The specific activity and Km values for both SSI and SSIIa were comparable between the bound and free forms (Table 2, Fig. 3A,B). This is consistent with the catalytic fold of SS enzymes being contained entirely within the Cterminal region of the primary sequence [7]. Further experiments were conducted with SSI, SSIIa, SSIII-HDCAT, and SSIII-CAT immobilized on S-agarose.

SSIII-HDCAT and SSIII-CAT (Fig. 1B) were also analyzed kinetically to determine specific activities and the Km for ADPGlc (Table 2, Fig. 3C). The specific activities of fulllength SSIII and the SSIII-HDCAT fragment could be compared directly in these assays because the purity of the two enzyme preparations was similar (Fig. 2). Truncated SSIII lacking the N domain, i.e., SSIII-HDCAT, displayed approximately one-fourth the specific activity of fulllength enzyme, although the Km value for ADPGlc was approximately the same for both forms (Table 2, Fig. 3C).

\subsection{Trans effects on enzymatic activity}

The observed difference in catalytic rate between full-length SSIII and the SSIII-HDCAT fragment suggested that the $\mathrm{N}$ domain influences catalytic activity. This was tested further by examining whether SSIII-N or SSIII-NHD can affect the activity of catalytic domains in trans, i.e., on separate molecules. Various SSIII fragments disconnected from the catalytic domain,

specifically SSIII-N, SSIII-NHD, or SSIII-HD were mixed with SSIII-CAT or SSIII-HDCAT in 
standard reaction conditions. As a negative control, the potential regulatory domain was also denatured by boiling and added to the catalytic fragment in a presumably inactive form. Comparing the apparent specific activity between assays including the denatured or native additions showed that SSIII-N stimulated SSIII-HDCAT activity in trans (Table 2, Fig. 4A), and SSIII-NHD similarly stimulated activity of SSIII-CAT domain (Table 2, Fig. 4B). Addition of HD alone did not affect SSIII-CAT activity (Table 2, Fig. 4C). Kinetic analysis revealed that the $\mathrm{Km}$ for ADPGlc was not changed appreciably by addition of SSIII-N or SSIII-NHD in trans (Table 2, Fig. 4A,B).

\subsection{Direct regulation between SSS}

The previous finding that loss of SSIII owing to mutation results in elevated total SS activity in endosperm extracts suggest that SSIII is a negative regulator of other SSs [13-15]. To test this hypothesis, SSI and full-length SSIII or SSIII fragments containing the catalytic domain were assayed together in a reconstituted mixture under conditions of substrate saturation. Known amounts of each component were included, either with both enzymes in non-denatured form or with one of the two enzymes inactivated by boiling. Thus, in all comparisons between assays the protein concentration was the same. Mixtures of SSI with SSIII-HDCAT or SSIII-CAT exhibited approximately the sum of the activities of the two enzymes assayed separately (Table 3). Mixture of SSI with full-length SSIII, in contrast, exhibited only $60 \%$ of the activity expected from the sum of the individual enzymes added to the assay. Neither SSIII-HD, SSIII-N, nor SSIII-NHD affected SSI activity in the reconstituted system (Table 3). Thus, there appears to be a negative regulatory interaction between SSI and SSIII that requires all three domains of the latter enzyme.

The same approach was used to test for regulatory interaction between SSI and SSIIa. In this instance the activity when the two enzymes were mixed matched the sum of the individual 
activities. SSI and SSIIa, thus act independently of each other (Table 3). This experiment also served to confirm that substrate was not limiting in these assay conditions. Finally, full-length SSIII and SSIIa were tested in the reconstituted system. Total activity was reduced to $85 \%$ of the amount predicted from the sum of the two individual enzymes (Table 3).

\subsection{Temperature stability}

SS has been proposed, based on enzymatic and flux analyses, as a step in starch metabolism in developing cereal grains that is particularly sensitive to growth at elevated temperature [20-22, 36]. Availability of purified recombinant proteins afforded the ability to individually test SSI, SSIIa, and SSIII for thermal inactivation during incubation at elevated temperatures likely to be encountered in field conditions. Each purified enzyme was incubated at a series of temperatures from $37^{\circ} \mathrm{C}$ to $50^{\circ} \mathrm{C}$ for up to $40 \mathrm{~min}$ prior to assay at $30^{\circ} \mathrm{C}$ reaction temperature. Fig. 5 shows a representative complete data set. Pre-incubation at up to $45^{\circ} \mathrm{C}$ had no effect on the activity of SSI. SSIIa, rather than displaying sensitivity elevated temperatures, appeared to stimulated by pre-incubation at $37^{\circ} \mathrm{C}$ to $45^{\circ} \mathrm{C}$. SSIII activity was stable during pre-incubation at $37^{\circ} \mathrm{C}$, but was sensitive to pre-incubation at $42^{\circ} \mathrm{C}$ or $45^{\circ} \mathrm{C}$ with loss of up to $50 \%$ of its activity. At the extreme preincubation temperature of $50^{\circ} \mathrm{C}$ all of the tested enzymes completely lost activity within $40 \mathrm{~min}$, although SSI appeared to be more resistant to this temperature than SSIIa or SSIII. Independent biological replications starting from separate E. coli cultures yielded essentially identical results showing stability of SSI during pre-incubation at elevated temperature, as well as for stimulation of SSIIa activity, and loss of activity of SSIII (data not shown).

These results are in agreement with previous studies in which recombinant SSI or SSIIa, or native SSIII purified from maize endosperm extracts, were assayed at increasing reaction temperatures [31-33]. Together the data indicate that all three SS enzymes are subject to thermal 
inactivation at lower temperatures than are typical for most enzymes. SSIII in particular loses activity at temperatures that are encountered in agricultural settings.

\subsection{Potential buffer effects on SS activity}

Potential effects of the buffer molecules used in SS assays were compared. A previous report suggested that bicine or Tris buffer molecules typically used for SS reactions sequester ADPGlc and thus artificially inhibit the observed enzyme activity [26]. Further, addition of glucans to the reaction was proposed to release the sequestered ADPGlc from its interaction with the buffer molecule. This proposal suggested that the well documented stimulation of in vitro SS activity by glucans resulted from this effect on ADPGlc levels rather than owing to the glucan participating directly as a glycosyl unit acceptor substrate. Glycine buffers were proposed to fail to sequester ADPGlc and thus should not exhibit stimulation of the SS reaction by glucans [26]. To test this hypothesis, the same preparation and quantity of recombinant full-length SSIII was assayed in two reaction conditions that varied only by the buffer molecule. Significant differences in Vmax were not observed between reactions containing either glycine or Tris buffer (Table 4). Inclusion of glycogen in the reaction stimulated activity 3- to 4-fold, and this was observed both with the Tris and the glycine buffer. Agarose-bound SSIIa was also used to compare potential buffer effects, in this instance comparing glycine and bicine buffers. Again the buffer in the reaction had no significant effect on Vmax either in the presence or absence of glycogen (Table 4), and 3-fold stimulation by addition of glucan was evident in both conditions. These results are inconsistent with the previous proposal [26], and favor the accepted mechanism in which exogenous glucan molecules in the reaction act directly as acceptor substrates of the SS. 


\section{Discussion}

\subsection{Interactions between SSs}

Understanding how starch is synthesized, in particular the achievement of crystallizationcompetent architecture in branched glucan polymers, will require defining the regulatory interactions that take place between components of the assembly system. This study adds to the body of evidence supporting the hypothesis that the multiple, conserved SSs necessary for normal amylopectin synthesis act in concert owing at least in part to direct associations between them. SSIII plays a central role in such coordination and in this study some of these functions were demonstrated to result from direct interactions. Considering the current study and previous results, SSIII has been shown to comprise numerous separable functions including 1) substrate binding at the active site, 2) glucan binding outside of the active site mediated by SSIII-HD [9], 3) protein-protein interactions mediated by SSIII-HD [11, 12], 4) a positive function of the SSIII$\mathrm{N}$ domain that stimulates activity of the SSIII catalytic domain, and 5) negative regulatory effects between SSIII and SSI, and potentially between SSIII and SSIIa.

A stimulatory effect of the maize SSIII-N domain was indicated by comparing the activities of full-length SSIII and the SSIII-HDCAT truncation product that lacks SSIII-N. This was confirmed by the results that the SSIII-N domain added in trans increased the in vitro activity of SSIII-HDCAT and that the combined SSIII-NHD domains when added to the catalytic domain SSIII-CAT had the same effect. Apparently both SSIII-N and SSIII-HD are required for full activation of SSIII-CAT, because when SSIII-HD alone was added to the catalytic domain no increase in the rate of catalysis was observed. The fact that the positive effects were observed when fragments were mixed in trans is consistent with a direct regulatory role resulting from contact between the domains, as opposed to an effect on folding or other maturation of the 
catalytic domain during biogenesis of the enzyme. A previous study of Arabidopsis SSIII compared activities between the full length enzyme and the catalytic domain when it was disconnected from SSIII-HD [9]. A stimulatory effect of SSIII-HD was demonstrated in that instance. The dicot species in comparison to maize, however, lacks the SSIII-N domain that is present in cereal species. The current study extends the observations on Arabidopsis SSIII by demonstrating that the SSIII-N from maize also has a positive effect on the enzyme's catalytic activity.

The total activity SSI and SSIII mixed together in a reconstituted system was substantially less than the sum of the two enzymes acting individually in exactly the same conditions. This observation is consistent with previous results demonstrating interaction between SSI and SSIII by co-immunoprecipitation, pull-down from amyloplast lysates, and in vivo protein-protein interaction assays $[11,12]$. These previous results showed that discrete regions of SSIII, including part of the N domain, or SSIIIHD, can independently bind to SSI. This is consistent with the finding in the current study that full-length SSIII was required for the negative interaction between that enzyme and SSI, so precisely which domains of SSIII are directly responsible for this effect cannot yet be discerned. Previous in vivo protein-protein interaction tests revealed that SSI binds SSIII-N without the participation of SSIII-HD, yet pull-down experiments demonstrated an interaction between SSI and SSIII-HD [11]. Further analyses will be required to determine the mechanism by which these multiple interactions between SSI and SSIII can control total enzyme activity. From the experiments in the current study it is not possible to determine which enzyme is negatively regulated when SSI and SSIII are mixed. SSIII could negatively regulate SSI, or visa versa, or there could be reciprocal regulation between the 
two components in the reconstituted system. Genetic analyses, however, showed that elimination of SSIII by mutation results in elevated total SS activity in endosperm cell extracts [13-15].

SSIII and SSIIa also displayed a negative interaction, although this effect was quantitatively less pronounced than that observed between SSIII and SSI. SSI and SSIIa, in contrast displayed fully additive activities when mixed, suggesting they act completely independently of each other. The common feature of the negative interaction is SSIII, so taken together, the in vivo and in vitro data support the conclusion that SSIII exerts a negative effect on the activity of SSI and potentially of SSIIa as well.

Further structural and enzymatic characterization will be required to understand the molecular mechanism(s) underlying the effects of the SSIII-N domain. Regarding the SSI-SSIII interaction, both proteins possess an $\mathrm{N}$ terminal domain that appears to regulate the activity of their own catalytic folds. Crystallized SSI from barley that contains both the conserved catalytic domain and the $\mathrm{N}$ terminal extension yielded detectable electron density only in the CAT domain fold [7]. Coupled with the observations that truncated SSI from either barley or maize exhibits elevated activity relative to the full-length enzyme [7, 31], the data suggest that the SSI N terminal extension may function to restrict access to the active site and can undergo molecular motions so as to eliminate such steric hindrance. In contrast to this negative function, the SSIII-N domain has a positive regulatory effect on the SSIII catalytic domain.

\subsection{SS thermosensitivity}

SS activity has been proposed as a temperature-sensitive step in cereal endosperm starch biosynthesis such that grain yield is reduced in high-temperature environments [20, 21]. Total SS activity in maize endosperm extracts exhibits thermal inactivation during prolonged incubation at $40^{\circ} \mathrm{C}$ or higher prior to being returned to optimal temperatures for the assay [21], and similar 
effects were observed on SSs purified from other botanical sources [37]. Effects in this temperature range are atypical of most enzymes, which in general are not subject to thermal inactivation below $60^{\circ} \mathrm{C}$. Results with recombinant maize enzymes essentially recapitulated the cell extract data, with SSI, SSIIa, and SSIII all exhibiting complete inactivation following incubation at $50^{\circ} \mathrm{C}$. The three enzymes had differing sensitivities to heat treatment, with SSI the most resistant and SSIII exhibiting the fastest inactivation and sensitivity to temperatures as low as $42^{\circ} \mathrm{C}$. These observations may be relevant in future consideration of the role of SSs as yield factors during growth of maize or other grains in elevated temperature environments, and SSIII in particular could play a significant role in this agronomic property.

SSIIa appears to be stimulated by pre-incubation at elevated temperatures. This was observed repeatedly in independent assays in the pre-incubation temperature range of $37^{\circ} \mathrm{C}$ to $45^{\circ} \mathrm{C}$. Taken together these temperature stability experiments raise the possibility that amylopectin structure could vary in endosperm tissue developing at different temperature ranges. A caveat to this conclusion is that the recombinant SSIIa assayed here had a single amino acid change, Leu 534 to Pro, compared to the naturally occurring enzyme. The kinetic properties of the recombinant enzyme were consistent with those reported for other sources of SSII, so drastic changes in catalytic properties were not evident. Nonetheless, subtle effects such as activation by pre-incubation at elevated temperature potentially could have arisen from this substitution.

\subsection{Buffer effects on SS activity and implications for its mechanism}

A recent challenge to the presumed SS mechanism suggested that exogenous glucans are not direct substrates of the reaction [26]. In this view the enzyme is proposed to be self-glycosylating so that exogenous acceptor molecules are not required [38-40]. Contrary to these arguments, the fact that glycogen addition stimulated the activity of purified SSIII or SSI regardless of the 
buffer present in the reaction supports the mechanism first suggested by Leloir and colleagues in which exogenous glucans are direct substrates that accept the incoming glucosyl unit donated from ADPGlc [41]. Much remains to be learned, however, regarding the role of the glucan acceptor in the SS reaction. Current unknowns include the role of surface glucan binding sites remote from the active site, identified in barley SSI [7], and the reasons that SSI, SSII, and SSIII appear to have different substrate and product specificities. Ready availability of recombinant SSI, SSIIa, and SSIII will be a valuable tool in addressing such future questions.

\section{Acknowledgements}

Oligonucleotide synthesis and DNA sequence analysis was performed by the Iowa State University DNA Facility. This work was supported by awards 2010-65115-20376 and 201067003-30215 from the U.S. Department of Agriculture, National Institute for Food and Agriculture.

\section{References}

[1] S.C. Zeeman, J. Kossmann, A.M. Smith, Annu. Rev. Plant Biol. 61 (2010) 209-234.

[2] T.A. Hennen-Bierwagen, A.M. Myers, in: P. Becraft (Ed.), Seed Genomics, WileyBlackwell, Ames, IA, 2013, pp. 123-137.

[3] I.J. Tetlow, Seed Science Research 21 (2011) 5-32.

[4] P. Deschamps, H. Moreau, A.Z. Worden, D. Dauvillee, S.G. Ball, Genetics 178 (2008) 2373-2387.

[5] N. Fujita, Y. Nakamura, in: I. Tetlow (Ed.), Starch: Origins, Structure, and Metabolism, Society for Experimental Biology, London, 2012, pp. 115-140.

[6] P.L. Keeling, A.M. Myers, Annu. Rev. Food Sci. Technol. 1 (2010) 271-303.

[7] J.A. Cuesta-Seijo, M.M. Nielsen, L. Marri, H. Tanaka, S.R. Beeren, M.M. Palcic, Acta crystallographica. Section D, Biological crystallography 69 (2013) 1013-1025. 
[8] M. Gao, J. Wanat, P.S. Stinard, M.G. James, A.M. Myers, Plant Cell 10 (1998) 399-412.

[9] H.A. Valdez, M.V. Busi, N.Z. Wayllace, G. Parisi, R.A. Ugalde, D.F. Gomez-Casati, Biochemistry 47 (2008) 3026-3032.

[10] N. Palopoli, M.V. Busi, M.S. Fornasari, D. Gomez-Casati, R. Ugalde, G. Parisi, Proteins 65 (2006) 27-31.

[11] T.A. Hennen-Bierwagen, F. Liu, R.S. Marsh, S. Kim, Q. Gan, I.J. Tetlow, M.J. Emes, M.G. James, A.M. Myers, Plant Physiol. 146 (2008) 1892-1908.

[12] T.A. Hennen-Bierwagen, Q. Lin, F. Grimaud, V. Planchot, P.L. Keeling, M.G. James, A.M. Myers, Plant Physiol. 149 (2009) 1541-1559.

[13] G.W. Singletary, R. Banisadr, P.L. Keeling, Plant Physiol. 113 (1997) 293-304.

[14] H. Cao, J. Imparl-Radosevich, H. Guan, P.L. Keeling, M.G. James, A.M. Myers, Plant Physiol. 120 (1999) 205-216.

[15] N. Fujita, M. Yoshida, T. Kondo, K. Saito, Y. Utsumi, T. Tokunaga, A. Nishi, H. Satoh, J.H. Park, J.L. Jane, A. Miyao, H. Hirochika, Y. Nakamura, Plant Physiol. 144 (2007) 2009-2023.

[16] X. Zhang, A.M. Myers, M.G. James, Plant Physiol. 138 (2005) 663-674.

[17] N. Szydlowski, P. Ragel, S. Raynaud, M.M. Lucas, I. Roldan, M. Montero, F.J. Munoz, M. Ovecka, A. Bahaji, V. Planchot, J. Pozueta-Romero, C. D'Hulst, A. Merida, Plant Cell 21 (2009) 2443-2457.

[18] Q. Lin, B. Huang, M. Zhang, J.G. Rivenbark, X. Zhang, M.G. James, A.M. Myers, T.A. Hennen-Bierwagen, Plant Physiol. 158 (2012) 679-692.

[19] Z. Li, D. Li, X. Du, H. Wang, O. Larroque, C.L. Jenkins, S.A. Jobling, M.K. Morell, J. Exp. Bot. 62 (2011) 5217-5231.

[20] K. Denyer, C.M. Hylton, A.M. Smith, Australian J. Agricul. Res. 21 (1994) 783-789.

[21] P.L. Keeling, R. Banisadr, L. Barone, B.P. Wasserman, G.W. Singletary, Australian Journal of Plant Physiology 21 (1994) 807-827.

[22] P.L. Keeling, P.J. Bacon, J.S. Holt, Planta 191 (1993) 342-348.

[23] P.L. Keeling, J.R. Wood, R.H. Tyson, I.G. Bridges, Plant Physiol. 87 (1988) 311-319.

[24] R.C. Muchow, Field Crops Research 23 (1990) 145-158.

[25] N. Cheikh, R.J. Jones, Physiologia Plantarum 95 (1995) 59-66.

[26] R. Mukerjea, A. McIntyre, J. Robyt, Carbohydr. Res. 355 (2012) 28-34. 
[27] M.E. Knight, C. Harn, C.E. Lilley, H. Guan, G.W. Singletary, C. MuForster, B.P. Wasserman, P.L. Keeling, Plant J. 14 (1998) 613-622.

[28] O. Emanuelsson, H. Nielsen, G. von Heijne, Protein Sci. 8 (1999) 978-984.

[29] R.T. Raines, M. McCormick, T.R. Van Oosbree, R.C. Mierendorf, Methods Enzymol. 326 (2000) 362-376.

[30] E. Hochuli, W. Bannwarth, H. Döbeli, R. Gentz, D. Stüber, Nature Biotech. 6 (1988) 1321-1325.

[31] J.M. Imparl-Radosevich, P. Li, L. Zhang, A.L. McKean, P.L. Keeling, H. Guan, Arch. Biochem. Biophys. 353 (1998) 64-72.

[32] J.M. Imparl-Radosevich, D.J. Nichols, P. Li, A.L. McKean, P.L. Keeling, H. Guan, Arch. Biochem. Biophys. 362 (1999) 131-138.

[33] H. Cao, M.G. James, A.M. Myers, Arch. Biochem. Biophys. 373 (2000) 135-146.

[34] J.L. Ozbun, J.S. Hawker, J. Preiss, Plant Physiol. 48 (1971) 765-769.

[35] C.D. Boyer, J. Preiss, Plant Physiol. 67 (1981) 1141-1145.

[36] A.H. Rijven, Plant Physiol. 81 (1986) 448-453.

[37] K. Denyer, A.M. Smith, Planta 186 (1992) 609-617.

[38] R. Mukerjea, L. Yu, J.F. Robyt, Carbohydr. Res. 337 (2002) 1015-1022.

[39] R. Mukerjea, J.F. Robyt, Carbohydr. Res. 340 (2005) 245-255.

[40] R. Mukerjea, J.F. Robyt, Carbohydr. Res. 340 (2005) 2206-2211.

[41] L.F. Leloir, M.A. De Fekete, C.E. Cardini, J Biol Chem 236 (1961) 636-641. 


\section{Tables}

\section{Table 1}

Expressed proteins

\begin{tabular}{|c|c|c|c|c|}
\hline Product & $\operatorname{Tag}^{a}$ & Residues $^{b}$ & $\begin{array}{l}\text { Predicted } \\
\text { Mass }(k D a)\end{array}$ & Plasmid Sequence $^{c}$ \\
\hline SSI & $\mathrm{N}$ term. $\mathrm{S}$ & $39-640$ & 70 & $\begin{array}{l}\text { https://www.dropbox.com } / \mathrm{s} / 02 \mathrm{f} 87 \mathrm{zxpjwbwtgl} / \mathrm{p} \\
\text { ET29-NStagSSI.dna?dl=0 }\end{array}$ \\
\hline SSIIa & $\mathrm{N}$ term. $\mathrm{S}$ & $61-732$ & 77 & $\begin{array}{l}\text { https://www.dropbox.com/s/bw22drg2efnx } 119 / \mathrm{p} \\
\text { ET29-NStagSSIIa.dna?dl=0 }\end{array}$ \\
\hline SSIII-CAT & $\mathrm{N}$ term. $\mathrm{S}$ & $1226-1674$ & 51 & $\begin{array}{l}\text { https://www.dropbox.com } / \mathrm{s} / 591 \mathrm{u} 0 \text { ces } 1 \mathrm{i} 12 \mathrm{r} 9 \mathrm{f} / \mathrm{pE} \\
\text { T29-NStagSSIIICAT.dna?dl=0 }\end{array}$ \\
\hline SSIII-HDCAT & $\mathrm{N}$ term. $\mathrm{S}$ & $770-1674$ & 104 & $\begin{array}{l}\text { https://www.dropbox.com/s/2gwb06kipogbhp0/ } \\
\text { pET29-NStagSSIIIHDCAT.dna?dl=0 }\end{array}$ \\
\hline SSIII & $\mathrm{C}$ term. His & $90-1674$ & 180 & $\begin{array}{l}\text { https://www.dropbox.com/s/9c14w81bdmxbkvj/ } \\
\text { pET29-SSIIIFLCH.dna?dl=0 }\end{array}$ \\
\hline SSIII-NHD & $\mathrm{C}$ term. His & $90-1225$ & 130 & $\begin{array}{l}\text { https://www.dropbox.com/s/kwe3dhsml6xdey5/ } \\
\text { pET29-SSIIINtermHDCH.dna?dl=0 }\end{array}$ \\
\hline SSIII-N & $\mathrm{C}$ term. His & $90-769$ & 76 & $\begin{array}{l}\text { https://www.dropbox.com/s/1p3iyteion } 7 \text { nalk/pE } \\
\text { T29-SSIIINtermCH.dna?dl=0 }\end{array}$ \\
\hline SSIII-HD & $\mathrm{C}$ term. His & $770-1225$ & 55 & $\begin{array}{l}\text { https://www.dropbox.com/s/z5t6chy2etozx } 8 x / p \\
\text { ET29-SSIIIHDCH.dna?dl=0 }\end{array}$ \\
\hline
\end{tabular}

a "N term. S" indicates attachment of the S-tag sequence of pET29 followed by a thrombin cleavage site, located at the $\mathrm{N}$ terminus of the recombinant protein. This region comprises 27-30 residues. "C term. His" indicates the 6X His-tag, comprising 6 sequential His residues, located at the $\mathrm{C}$ terminus of the protein.

${ }^{b}$ Values indicate the $\mathrm{N}$ terminal and $\mathrm{C}$ terminal residues of the SS polypeptide included in the recombinant protein. Reference sequences for amino acid numbering are: SSI, Genbank accession AAB99957; SSIIa, Genbank accession BT084561; SSIII, gene model GRMZM2G14139 (www.maizeGDB.org).

${ }^{c}$ Annotated sequence files can be downloaded from the indicated web address and viewed with the free program SnapGene Viewer (http://www.snapgene.com/products/ snapgene_viewer/). 


\section{Table 2}

Enzymatic properties

\begin{tabular}{llcc}
\hline Enzyme & Status & $\begin{array}{c}\text { Specific activity } \\
(\mu \mathrm{mol} / \mathrm{min} / \mathrm{mg})\end{array}$ & $\begin{array}{c}\mathrm{Km}^{c} \\
(\mathrm{mM})\end{array}$ \\
\hline SSI & Bound & $5.01 \pm 0.41$ & $0.55 \pm 0.08$ \\
SSI & Free & 6.16 & 0.39 \\
SSIIa & Bound & $8.62 \pm 0.25$ & $0.73 \pm 0.06$ \\
SSIIa & Free & 9.75 & 0.84 \\
$\begin{array}{l}\text { SSIII full-length } \\
\text { SSIII-HDCAT }\end{array}$ & Free & $6.33 \pm 0.17$ & $0.33 \pm 0.00$ \\
SSIII-CAT & Bound & $1.60 \pm 0.02$ & $0.39 \pm 0.01$ \\
\hline $\begin{array}{l}\text { SSIII-HDCAT }+ \\
\text { native SSIII-N }\end{array}$ & Bound & $1.13 \pm 0.08$ & $0.49 \pm 0.05$ \\
$\begin{array}{l}\text { SSIII-HDCAT }+ \\
\text { denatured SSIII-N }\end{array}$ & Bound & $4.67 \pm 0.40$ & $0.64 \pm 0.10$ \\
\hline $\begin{array}{l}\text { SSIII-CAT }+ \\
\text { denatured SSIII-NHD }\end{array}$ & Bound & $2.69 \pm 0.32$ & $0.87 \pm 0.21$ \\
$\begin{array}{l}\text { SSIII-CAT }+ \\
\text { denatured SSIII-NHD }\end{array}$ & Bound & $4.65 \pm 0.55$ & $0.59 \pm 0.21$ \\
\hline $\begin{array}{l}\text { SSIII-CAT }+ \\
\text { native SSIII-HD }\end{array}$ & Bound & $1.95 \pm 0.37$ & $0.72 \pm 0.18$ \\
$\begin{array}{l}\text { SSIII-CAT }+ \\
\text { denatured SSIII-HD }\end{array}$ & Bound & $2.73 \pm 0.27$ & $1.01 \pm 0.17$ \\
\hline
\end{tabular}

a "Bound" indicates enzymes attached to S-agarose beads through the Stag/S-protein interaction, and "Free" indicates enzymes in solution.

${ }^{b} \mathrm{SS}$ activity at $30^{\circ} \mathrm{C}$ was determined from ADP released upon addition of glucosyl units from ADPGlc to a glycogen acceptor. Values with standard deviation are the average of three independent replicates, and those without standard deviation are from a single replicate.

${ }^{c} \mathrm{Km}$ and maximum velocity were calculated from the intercepts of double reciprocal plots. Values with standard deviation are the average of three independent replicates, and those without standard deviation are from a single replicate. 
Table 3

Reconstitution of SS mixtures

\begin{tabular}{|c|c|c|c|c|}
\hline \multicolumn{2}{|c|}{ Component 1} & \multicolumn{2}{|c|}{ Component 2} & \multirow{2}{*}{$\begin{array}{c}\text { Activity }^{a} \\
(\mu \mathrm{mol} / \mathrm{min} / \mathrm{mg})\end{array}$} \\
\hline Protein & Status & Protein & Status & \\
\hline SSI & native & SSIII & denatured & $3.36 \pm 0.0$ \\
\hline SSI & denatured & SSIII & native & $3.00 \pm 0.20$ \\
\hline SSI & native & SSIII & native & $3.78 \pm 0.14$ \\
\hline \multicolumn{2}{|c|}{ Predicted sum of activities } & & & 6.36 \\
\hline SSI & native & SSIII-HDCAT & denatured & $3.40 \pm 0.29$ \\
\hline SSI & denatured & SSIII-HDCAT & native & $0.78 \pm 0.03$ \\
\hline SSI & native & SSIII-HDCAT & native & $3.90 \pm 0.04$ \\
\hline \multicolumn{2}{|c|}{ Predicted sum of activities } & & & 4.18 \\
\hline SSI & native & SSIII-CAT & denatured & $3.31 \pm 0.08$ \\
\hline SSI & denatured & SSIII-CAT & native & $0.62 \pm 0.05$ \\
\hline SSI & native & SSIII-CAT & native & $3.82 \pm 0.17$ \\
\hline \multicolumn{2}{|c|}{ Predicted sum of activities } & & & 3.93 \\
\hline SSI & native & SSIII-HD & native & $3.52 \pm 0.08$ \\
\hline SSI & native & SSIII-HD & denatured & $3.50 \pm 0.09$ \\
\hline SSI & native & SSIII-N & native & $3.33 \pm 0.08$ \\
\hline SSI & native & SSIII-N & denatured & $3.46 \pm 0.38$ \\
\hline SSI & native & SSIII-NHD & native & $3.24 \pm 0.09$ \\
\hline SSI & native & SSIII-NHD & denatured & $3.42 \pm 0.06$ \\
\hline SSI & native & SSIIa & denatured & $3.64 \pm 0.28$ \\
\hline SSI & denatured & SSIIa & native & $3.16 \pm 0.38$ \\
\hline SSI & native & SSIIa & native & $6.87 \pm 0.11$ \\
\hline \multicolumn{2}{|c|}{ Predicted sum of activities } & & & 6.80 \\
\hline SSIIa & native & SSIII & denatured & $3.13 \pm 0.18$ \\
\hline SSIIa & denatured & SSIII & native & $3.10 \pm 0.11$ \\
\hline SSIIa & native & SSIII & native & $5.30 \pm 0.23$ \\
\hline \multicolumn{2}{|c|}{ Predicted sum of activities } & & & 6.23 \\
\hline
\end{tabular}

${ }^{a} \mathrm{SS}$ assays at $30^{\circ} \mathrm{C}$ were performed independently in triplicate. Values are the mean and standard deviation. 
Table 4

Comparison of SS activity in different buffers

\begin{tabular}{|c|c|c|c|}
\hline \multirow[t]{2}{*}{ Enzyme } & \multirow[t]{2}{*}{ Buffer } & \multicolumn{2}{|c|}{$\mathrm{Vmax}(\mathrm{pmol} \mathrm{ADP} / \mathrm{min})^{a}$} \\
\hline & & - glycogen & + glycogen \\
\hline SSIII & glycine & $52.1 \pm 10.5$ & $181.1 \pm 9.7$ \\
\hline SSIII & Tris & $47.7 \pm 12.3$ & $175.3 \pm 5.8$ \\
\hline SSIIa & glycine & $22.1 \pm 1.6$ & $63.1 \pm 2.0$ \\
\hline SSIIa & bicine & $23.9 \pm 0.2$ & $71.8 \pm 2.6$ \\
\hline \multicolumn{4}{|c|}{$\begin{array}{l}{ }^{a} \text { Reactions contained } 1 \mathrm{mM} \text { ADPGlc plus or minus } \\
10 \mathrm{mg} / \mathrm{mL} \text { glycogen. Other reaction conditions were the } \\
\text { same in all instances except for the buffering molecule. } \\
\text { SS reactions at } 30^{\circ} \mathrm{C} \text { were performed in triplicate. Values } \\
\text { are the mean and standard deviation. }\end{array}$} \\
\hline
\end{tabular}




\section{Figure captions}

Fig. 1. SS domains and expressed proteins. Figures are drawn to scale except for promoters and affinity tags. A, Maize enzymes predicted from cDNA sequences, with E. coli glycogen synthase (GS) included for comparison. GT_1 and GT_5 are conserved domains that together constitute the conserved GT-B fold of the catalytic domain of glucosyl transferases such as SS. Green boxes indicate linker sequences that connect GT_1 and GT_5. Gray boxes indicate known or predicted plastid transit peptides. Crosshatched boxes and the solid yellow box indicate $\mathrm{N}$ terminal extension regions of distinct sequence. B, Proteins expressed in E. coli. Domain designations are as in panel A with the additions that purple triangles indicate the phage $\mathrm{T} 7$ promoter and black boxes indicate affinity tags.

Fig. 2. Recombinant protein purification. Asterisks indicate intact recombinant proteins in the purified fractions. A, Purification based on the C-terminal 6X His tags. Total soluble E. coli extracts from cells grown without induction of the T7 promoter (- IPTG) or after induction (+ IPTG), and purified proteins eluted from $\mathrm{Ni}^{++}$-agarose affinity matrix were separated by SDSPAGE and stained with Coomassie Blue. B, Purification based N terminal S-tags. Purified fractions are from S-protein agarose affinity matrix that was boiled to release bound proteins. TE, total extract. Asterisks indicate intact recombinant proteins in the purified fractions.

Fig. 3. Kinetic analysis of individual enzyme activities. All assays included $10 \mathrm{mg} / \mathrm{mL}$ glycogen, and ADPGlc was varied as indicated. SS activity was determined at $30^{\circ} \mathrm{C}$. "Bound" indicates the enzyme remains attached to S-agarose beads, and "Free" indicates the enzyme has been removed from the beads by cleavage with thrombin. Values are the average of three independent assays, 
and symbols colored in red are calculations from the best fit linear equation of the data points. Summary data are presented in Table 2. A, SSI activities. B, SSIIa activities. C, SSIII activities.

Fig. 4. Kinetic analyses of SSIII catalytic fragments mixed with N-terminal domain fragments. All assays included $10 \mathrm{mg} / \mathrm{mL}$ glycogen, and ADPGlc was varied as indicated. SS activity was determined at $30^{\circ} \mathrm{C}$. Values are the average of three independent assays, and symbols colored in red are calculations from the best fit linear equation of the data points. Summary data are presented in Table 2. A, SSIII-HDCAT reconstituted with native or denatured SSIII-N. B, SSIII-CAT reconstituted with native or denatured SSIII-NHD. C, SSIII-CAT reconstituted with native or denatured SSIII-HD.

Fig. 5. Effects of pre-incubation at elevated temperature on SS activity. Recombinant SSs were pre-incubated for various times at the indicated temperature, then specific activity was determined in conditions of substrate saturation at the assay temperature of $30^{\circ} \mathrm{C}$. The results of one representative experiment are shown. 


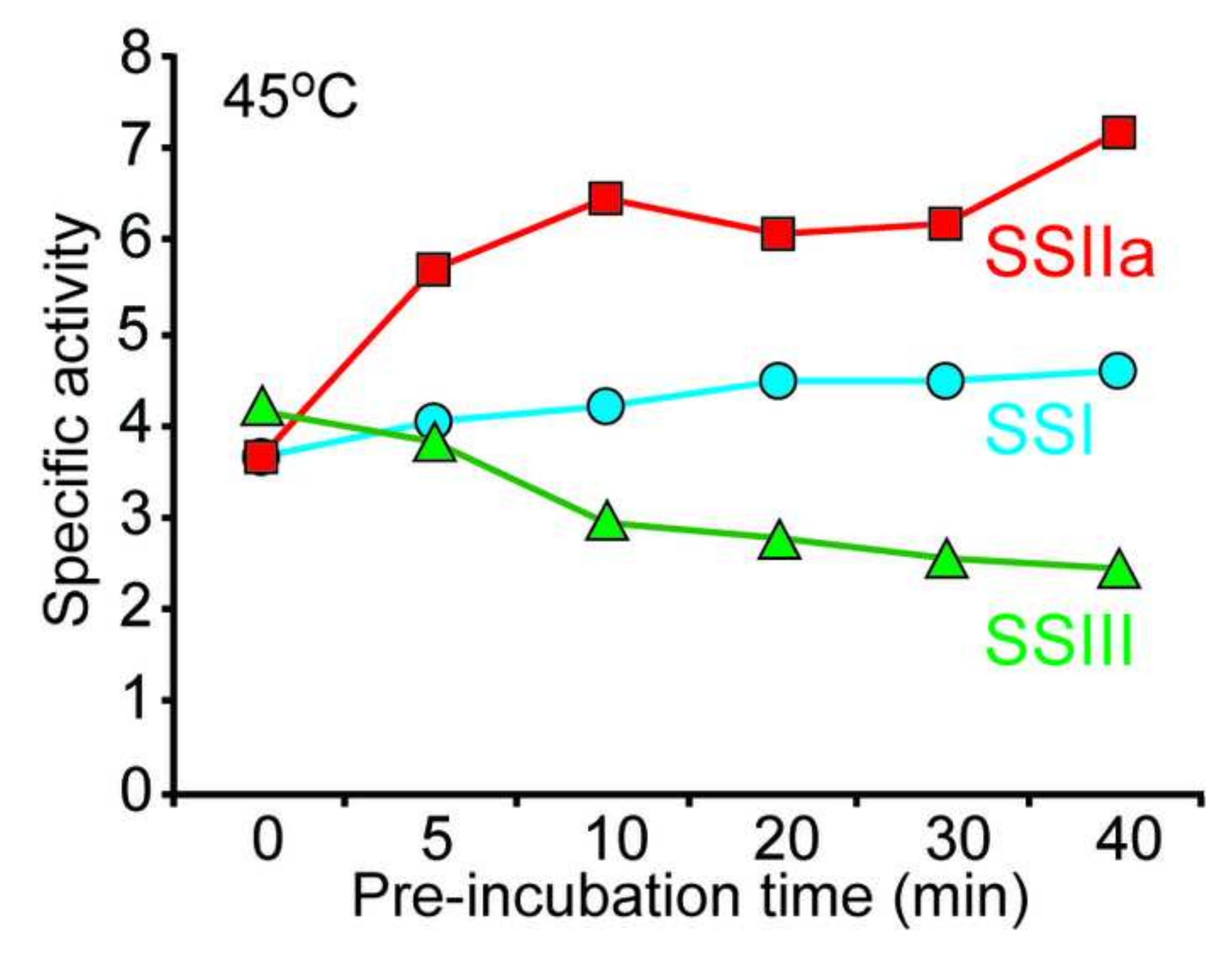

.
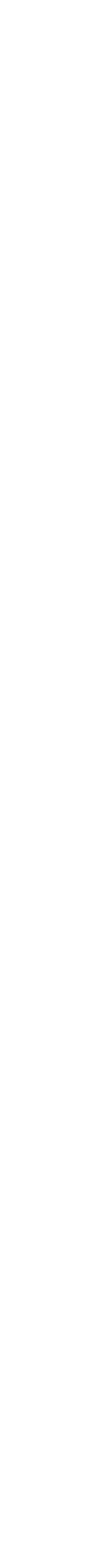


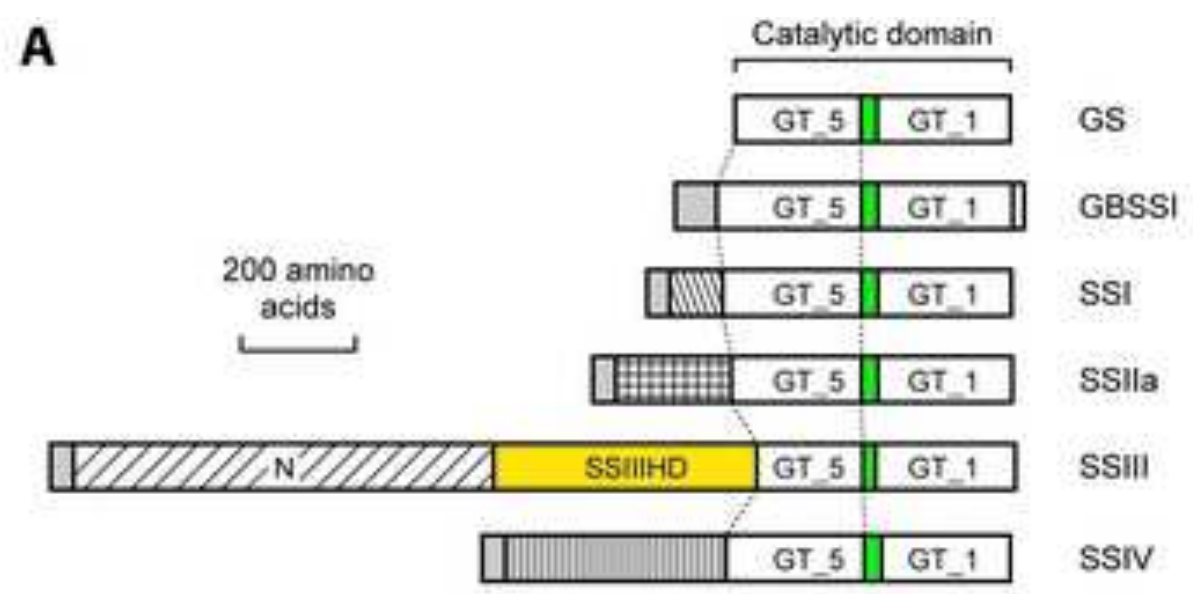

B

\begin{tabular}{|c|c|c|}
\hline \multirow{2}{*}{ DUDIINDIDIA } & 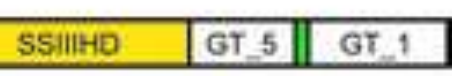 & ssill full-length \\
\hline & \begin{tabular}{|l||l|} 
GT_5 & GT_1 \\
\end{tabular} & SSIILHDCAT \\
\hline & NG.5I|GI-1 & SSIII-CAT \\
\hline ZIIIINDZDZDA & SSIIIHO & SSIII-NHD \\
\hline DIIIDCN & & SSIIIN \\
\hline & SSIIHD & SSIII-HD \\
\hline 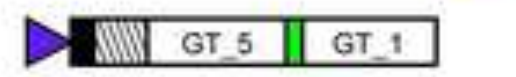 & & ssi \\
\hline 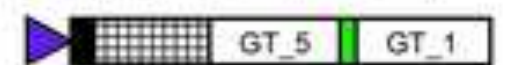 & & ssila \\
\hline
\end{tabular}

Fig. 1. SS domains and expressed proteins. Figures are drawn to scale except for promoters and affinity tags. A, Maize enzymes predicted from cDNA sequences, with $E$. coli glycogen synthase (GS) included for comparison. GT_1 and GT_5 are conserved domains that together constitute the conserved GT-B fold of the catalytic domain of glucosyl transferases such as SS. Green boxes indicate linker sequences that connect GT_1 and GT_5. Gray boxes indicate known or predicted plastid transit peptides. Crosshatched boxes and the solid yellow box indicate $\mathrm{N}$ terminal extension regions of distinct sequence. B, Proteins expressed in E. coli. Domain designations are as in panel $\mathrm{A}$ with the additions that purple triangles indicate the phage $\mathrm{T} 7$ promoter and black boxes indicate affinity tags. 

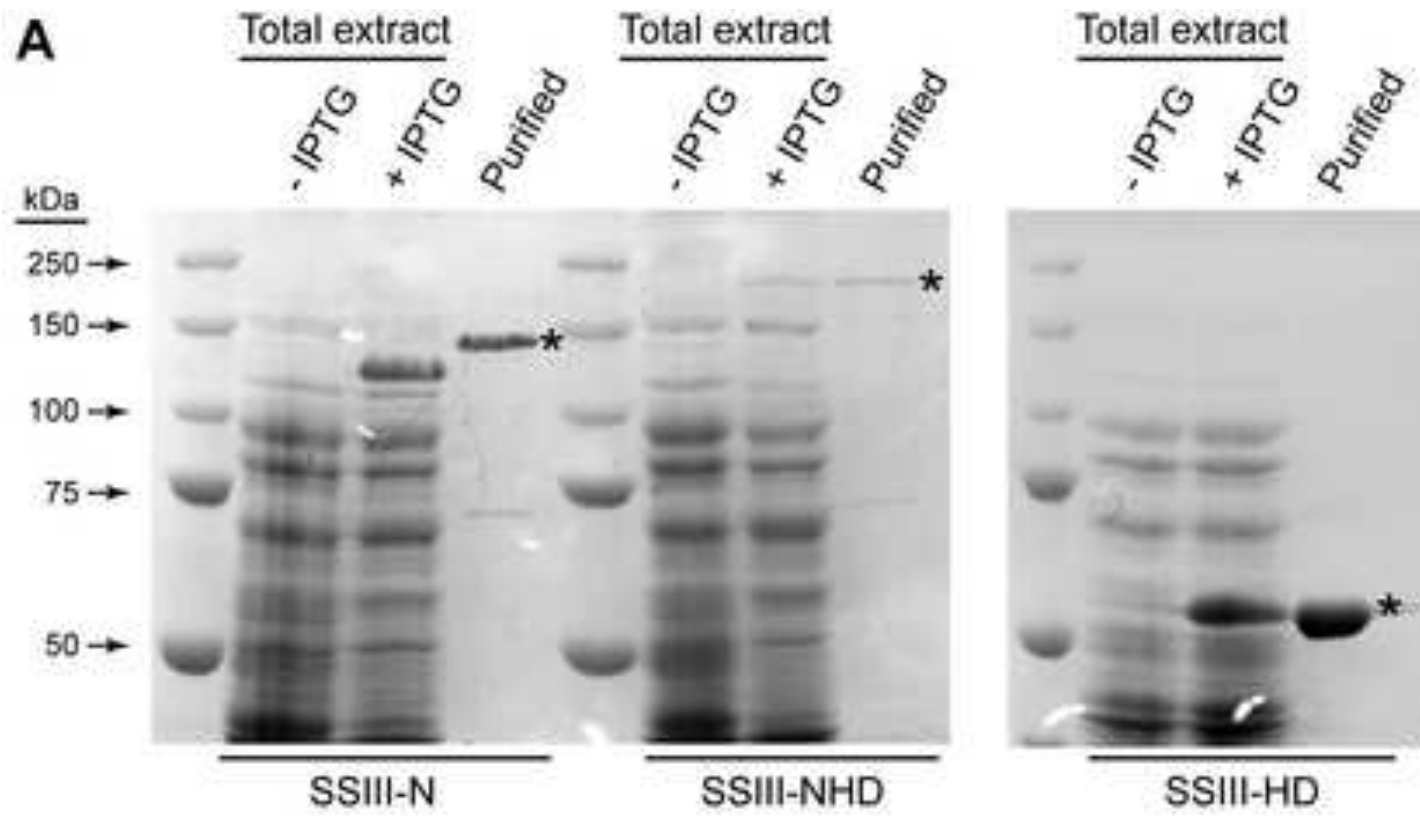

Total extract
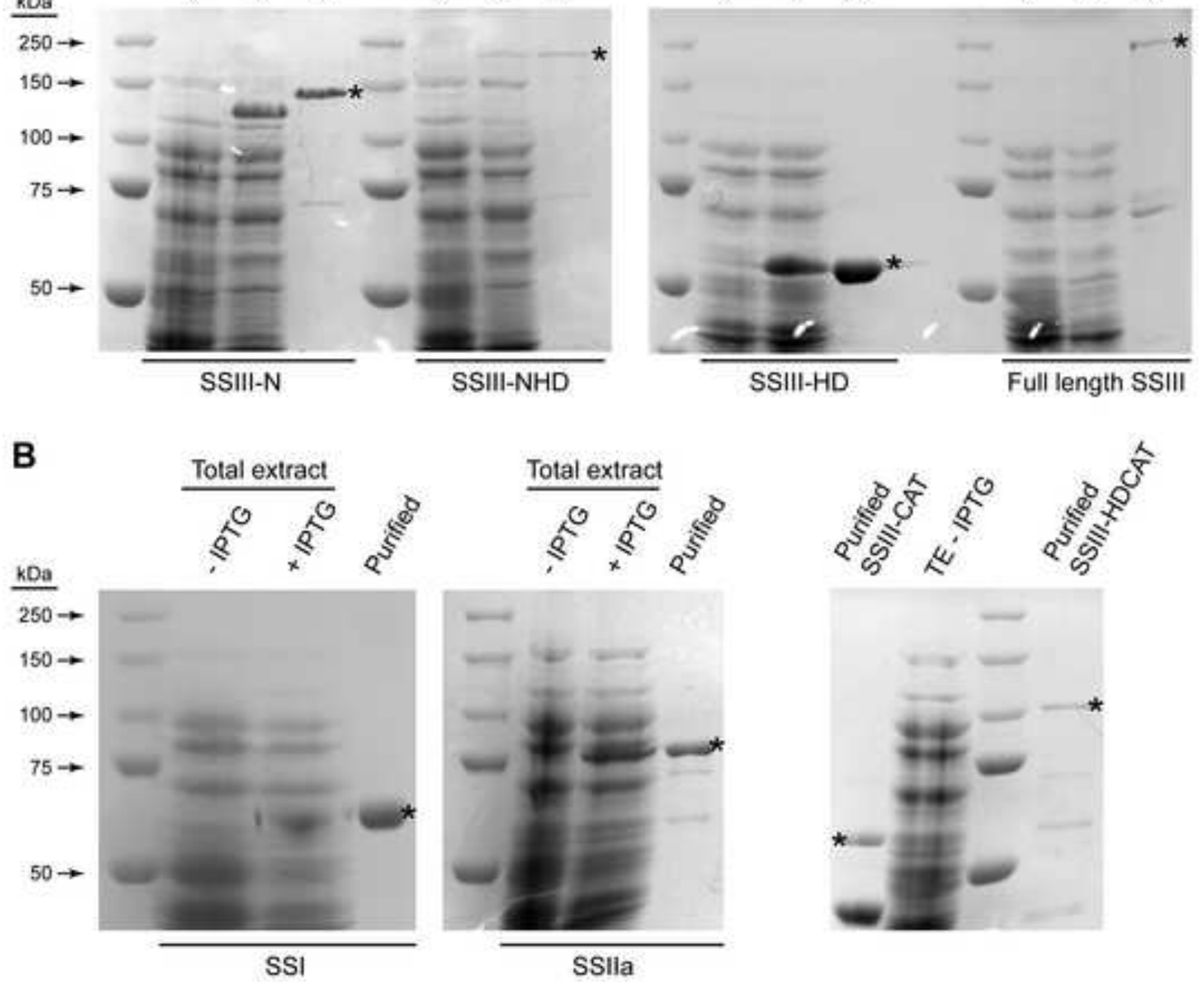

Fig. 2. Recombinant protein purification. A, Purification based on $\mathrm{C}$-terminal $6 \mathrm{X}$ His tags. Total soluble E. coli extracts from cells grown without induction of the T7 promoter (- IPTG) or after induction (+ IPTG), and purified proteins eluted from $\mathrm{Ni}^{++}$-agarose affinity matrix were separated by SDS-PAGE and stained with Coomassie Blue. B, Purification based on $\mathrm{N}$ terminal S-tags. Purified fractions are from S-protein agarose affinity matrix that was boiled to release bound proteins. TE, total extract. Asterisks indicate intact recombinant proteins in the purified fractions. 

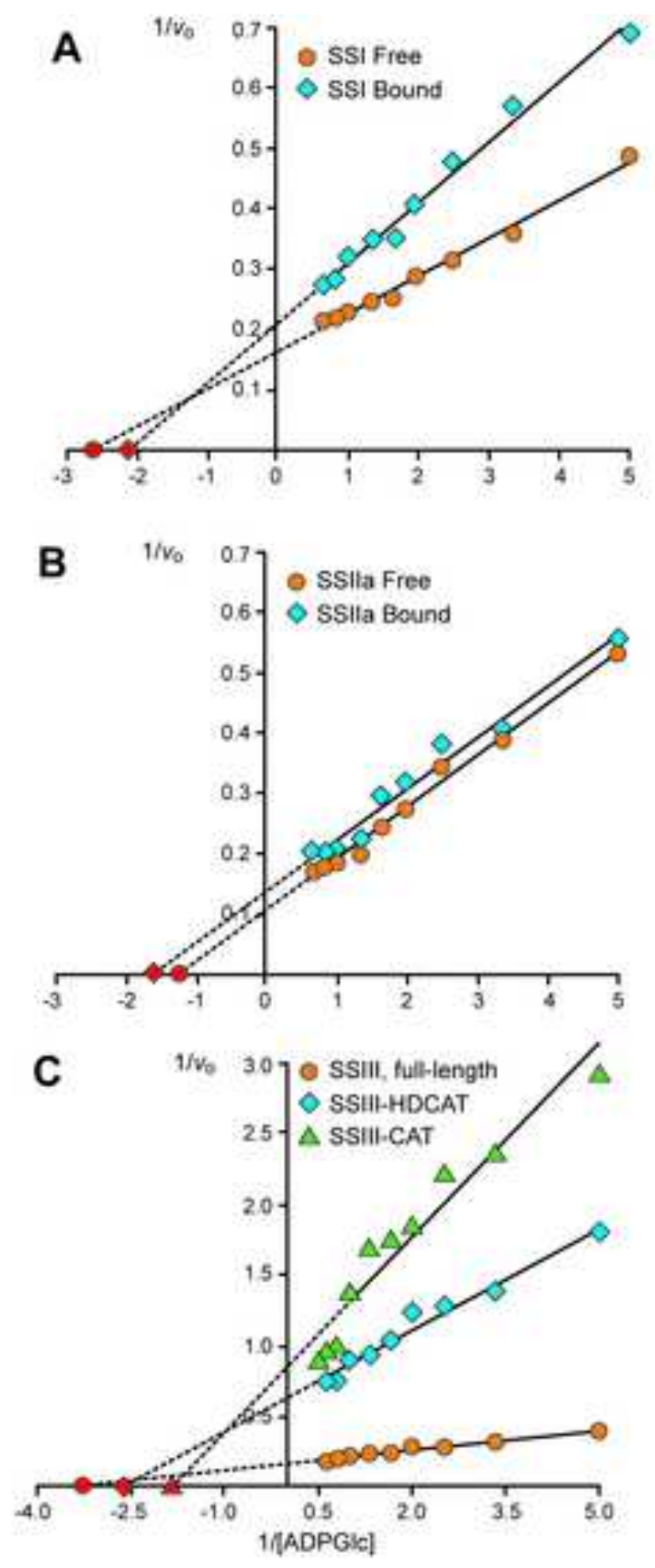

Fig. 3. Kinetic analysis of individual enzyme activities. All assays included $10 \mathrm{mg} / \mathrm{mL}$ glycogen, and ADPGlc was varied as indicated. SS activity was determined at $30^{\circ} \mathrm{C}$. "Bound" indicates the enzyme remains attached to S-agarose beads, and "Free" indicates the enzyme has been removed from the beads by proteolyic cleavage. Values are the average of three independent assays, and symbols colored in red are calculations from the best fit linear equation of the data points. Summary data are presented in Table 2. A, SSI activities. B, SSHa activities. C, SSIII activities. 

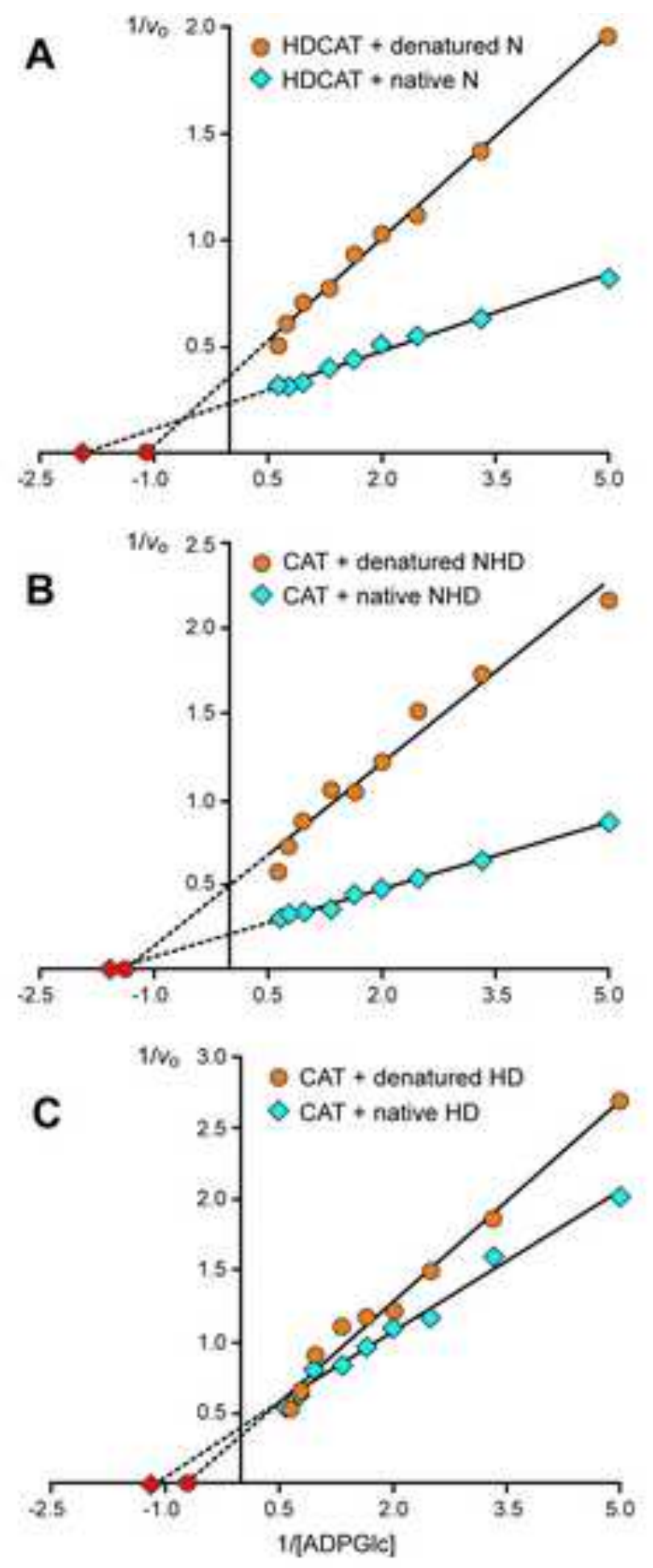

Fig. 4. Kinetic analyses of SSIII catalytic fragments mixed with $\mathrm{N}$-terminal domain fragments. All assays included $10 \mathrm{mg} / \mathrm{mL}$ glycogen, and ADPGle was varied as indicated. SS activity was determined at $30^{\circ} \mathrm{C}$. Values are the average of three independent assays, and symbols colored in red are calculations from the best fit linear equation of the data points. A. SSIII-HDCAT reconstituted with native or denatured SSIII-N, B, SSIII-CAT reconstituted with native or denatured SSIII-NHD. C, SSIII-CAT reconstituted with native or denatured SSIII-HD. 

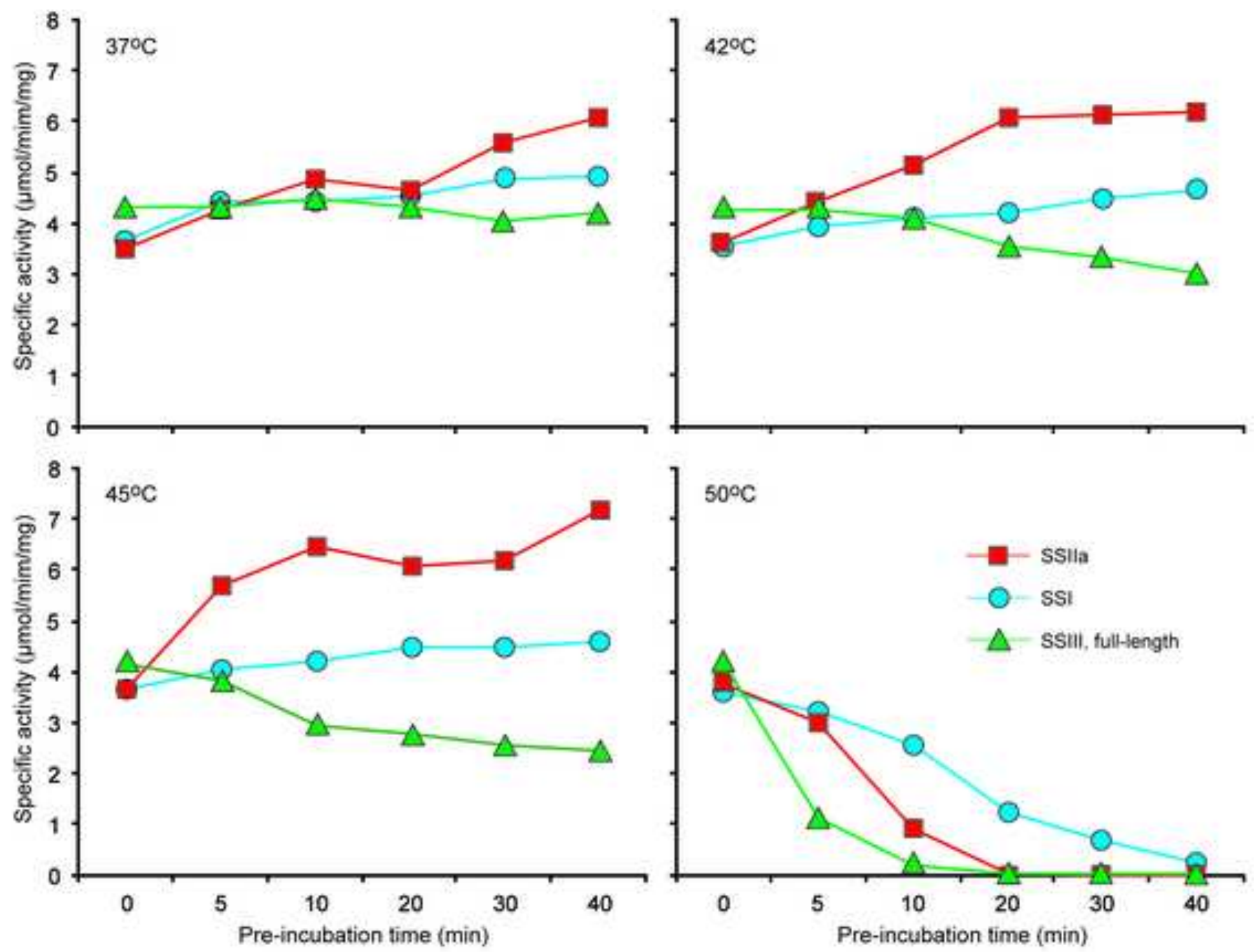

Fig. 5. Effects of pre-incubation at elevated temperature on SS activity. Recombinant SSs were pre-incubated for various times at the indicated temperature, then specific activity was determined in conditions of substrate saturation at the assay temperature of $30^{\circ} \mathrm{C}$. The results of one representative experiment are shown. 